\title{
Effect of Primary Tumour Resection on Patients Survival in Stage IV Breast Cancer
}

\author{
Timucin Erol ${ }^{1}$, Ersin Gurkan Dumlu ${ }^{2^{\star}}$ and Atac Baykal ${ }^{3}$ \\ ${ }^{1}$ Turkish Religious Foundation Private 29, Mayis Hospital, Ankara, Turkey. \\ ${ }^{2}$ Department of General Surgery, Ataturk Research and Training Hospital, Ankara, Turkey. \\ ${ }^{3}$ Department of General Surgery, Hacettepe University Faculty of Medicine, Ankara, Turkey.
}

Authors' contributions

All authors participate in designation of the study, managing the literature search, performing the statistical analysis, writing of the protocol, and the first draft of the manuscript. All authors read and approved the final manuscript.

Research Article

Received $3^{\text {rd }}$ May 2013

Accepted $27^{\text {th }}$ June 2013

Published $4^{\text {th }}$ July 2013

\section{ABSTRACT}

Aims: We aimed to evaluate the effect of primary tumour resection in stage IV breast cancer on survival.

Study Design: Retrospective data analysis.

Place and Duration of Study: Hacettepe University Faculty of Medicine Departments of General Surgery and Medical Oncology between 2001 and 2008.

Methodology: A total of 118 patients with breast cancer in whom metastasis was found at the time of diagnosis or within two months of operation were included in the present study and analysed retrospectively. Seventy-six patients $(63.4 \%)$ underwent surgical treatment, while $42(36.6 \%)$ underwent only diagnostic biopsy and were administered medical treatment.

Results: The mean follow-up was 28 months. The median overal survival was 44 months in operation group and 34 months in non-operation group. The oestrogen and progesterone receptor status, and type of metastasis had a significant effect on survival. The median two-year survival was higher in operation group (23 months vs. 18 months, $p=0.013)$. The survival was significantly higher in patients with only bone metastasis than those with other types of metastasis $(p<0.001)$. Among patients with only bone metastasis, there was no survival difference between operation and non-operation groups $(p=0.201)$. The median survival was significantly longer in operation group than 
non-operation group in patients with only visceral metastasis $(p=0.006)$.

Conclusion: The surgical treatment has favorable effect on survival in stage IV breast cancer, thus should be combined with systemic treatment to increase success rates.

Keywords: Breast neoplasms; surgical procedures; operative; neoplasm metastasis; tumor; survival.

\section{INTRODUCTION}

Breast cancer is the most common cancer among women and is the second leading cause of death from cancer after lung cancer [1]. Its incidence in the United States is 123/100,000, and around 40,000 people lose their lives annually due to invasive breast cancer [1]. The incidence of breast cancer has increased over the years owing to prolongation of expected duration of life, changes in lifestyle, and decreased numbers of deaths due to other reasons. In spite of this increase, mortality associated with breast cancer has decreased because of early diagnosis and advances in treatment methods. Despite all of these advances in diagnosis and treatment, patients at advanced stages at the time of diagnosis account for approximately $4 \%$ of all patients, and their expected rate of survival is low [1].

Treatment methods that should be used in such patients have become a matter of debate. With the publication of inoperability criteria for breast cancer by Haagensen and Stout in 1943, surgery was considered to be a palliative treatment in patients with stage IV breast cancer in whom the tumour is symptomatic, such as in cases of infection, bleeding, and ulceration [2]. However, more recent studies have established that removal of the main tumour in patients with stage IV breast cancer has a positive effect on survival and suggested that these patients may benefit from surgical treatment [3].

The aim of the present study was to establish whether surgical treatment of the primary tumour in patients with stage IV breast cancer at the time of diagnosis has any effect on survival and to determine the patient population that could benefit from such treatment.

\section{MATERIALS AND METHODS}

\subsection{Population}

A total of 118 patients who were diagnosed and treated in the Hacettepe University Faculty of Medicine Departments of General Surgery and Medical Oncology between 2001 and 2008 and in whom metastasis was found at the time of diagnosis or within two months of operation were included in the present study and analyzed retrospectively. Seventy-six patients $(63.4 \%)$ underwent surgical treatment (operation group), while 42 patients $(36.6 \%)$ underwent only diagnostic biopsy and were administered medical treatment including chemotherapy and hormonal therapy (non-operation group) on patients' request.

The study was approved by the Institutional Ethics Committee, and informed consent was waived for the retrospective design of the study. 


\subsection{Data Collection and Evaluation}

Records of the patients were evaluated retrospectively, and age, menopausal status, comorbid diseases, diagnostic method employed, number of metastases, metastasis region, hormone receptor status, whether surgical treatment was performed, tumour size in patients undergoing surgical treatment, and survival status as of 2009 were recorded.

\subsection{Statistical Analysis}

Statistical analysis was performed using the Statistical Package for Social Sciences (SPSS) version 15.0 software (SPSS Inc., Chicago, IL, US). In terms of descriptive statistics, age was expressed as the mean \pm standard deviation, and categorical variables were expressed as the number of cases and percentage. The significance of the difference in mean age between the operation group and the non-operation group was evaluated by Student's t test. Categorical variables were evaluated with Pearson's chi-square test. Whether categorical variables had a significant effect on overall survival was evaluated by Kaplan-Meier survival analysis using the log-rank test. The crude survival rate for subgroups of each variable and the mean expected survival and its $95 \%$ confidence interval $(\mathrm{Cl})$ were calculated. The effects of risk factors that had a significant effect on overall survival were investigated using in univariate analysis. The effect of each risk factor on mortality was investigated using $95 \%$ confidence intervals and significance levels. A $p$ value $<0.05$ was considered to indicate statistical significance.

\section{RESULTS AND DISCUSSION}

\subsection{Characteristics of Study Population}

The mean age of the 118 patients included in the study was $52.3 \pm 11$ years. A total of $33.3 \%$ of the patients were premenopausal. It was established that the most frequent diagnostic method was incisional biopsy $(68.6 \%)$. The most common pathological diagnosis was infiltrative ductal carcinoma $(70.1 \%)$. The demographic and tumor characteristics of patients in operation and non-operation groups are summarized in Table 1. No significant difference was found with regard to age, tumour degree, and oestrogen receptor and progesterone receptors staining between operation and non-operation groups. However, there was a significant difference between the two groups in terms of the number and type of metastases. It was established that in patients with metastasis in only one region, surgical treatment was performed more commonly; $68.4 \%$ of the patients in the surgical treatment group had a single metastasis. However, the large majority of patients who did not undergo surgical treatment had more than one metastasis. Thus, the number of metastatic regions and rate of multiple metastasis was significantly more common in non-operation group ( $p=$ 0.007 and $p=0.031$, respectively). 
Table 1. Demographic and tumor characteristics of operation and non-operation groups

\begin{tabular}{lllll}
\hline & & $\begin{array}{l}\text { Operation } \\
\text { group } \\
(\mathbf{n = 7 6 )}\end{array}$ & $\begin{array}{l}\text { Non-operation } \\
\text { group } \\
(\mathbf{n = 4 2 )}\end{array}$ & $\begin{array}{l}\boldsymbol{p} \\
\text { value }\end{array}$ \\
\hline Age (years) & 1 & $51.1 \pm 11.8$ & $54.5 \pm 11.4$ & 0.990 \\
Grade & 2 & $1(1.5 \%)$ & $2(4.8 \%)$ & 0.218 \\
& 3 & $33(50.8 \%)$ & $10(23.8 \%)$ & \\
Oestrogen receptor & $(+)$ & $31(47.7 \%)$ & $16(38.1 \%)$ & 1.000 \\
Progesterone receptor & $(-)$ & $51(67.1 \%)$ & $29(69 \%)$ & \\
The number of & $(-)$ & $21(27.6 \%)$ & $11(26.2 \%)$ & 0.910 \\
metastatic regions & 1 & $44(57.9 \%)$ & $26(61.9 \%)$ & \\
& 2 & $27(35.5 \%)$ & $14(14.4 \%)$ & 0.007 \\
Metastasis sites & 3 & $52(68.4 \%)$ & $18(42.9 \%)$ & \\
& Only bone & $20(26.4 \%)$ & $15(35.7 \%)$ & \\
& Single metastasis & $23(30.3 \%)$ & $8(19.9 \%)$ & 0.031 \\
& (extraosseous, & & \\
& visceral) & & \\
& Multiple metastasis & $25(25 \%)$ & $23(54.8 \%)$ & \\
\hline
\end{tabular}

\subsection{Survival and Factors Affecting Overall Survival}

The mean duration of follow-up was 28 months. The median duration of overal survival was 44 months in operation group and 34 months in non-operation group. The following factors had no effect on survival: age, menopausal status, comorbidity, grade, number of metastases, and operation type. On the other hand, the oestrogen receptor and progesterone receptor status and the type of metastasis were related to survival.

\subsection{Effects of Surgery and Bone Metastasis on Two-Year Survival}

In the evaluation of two-year survival, the median survival in the operation group was 23 months, while it was 18 months in the group that did not undergo operation $(p=0.013)$ (Fig. 1).

The expected median survival in patients with only bone metastasis was 63.8 months $(95 \%$ $\mathrm{Cl}, 53.0-73.6$ months), while that of patients with other types of metastasis was 34.2 months $(95 \% \mathrm{Cl}, 28.8-39.6$ months); this difference was statistically significant $(p<0.001)$ (Fig. 2). When patients with only bone metastasis were evaluated in both groups, no significant difference was found between the operation group and the non-operation group in terms of median survival $(p=0.201)$. In the evaluation of two-year survival in patients with visceral metastasis, the median survival was found to be 23 months $(95 \% \mathrm{Cl}, 18.1-27.8)$ in the operation group and 17 months $(95 \% \mathrm{Cl}, 3.3-30.6)$ in the non-operation group with a statistically significant difference $(p=0.006)$. 


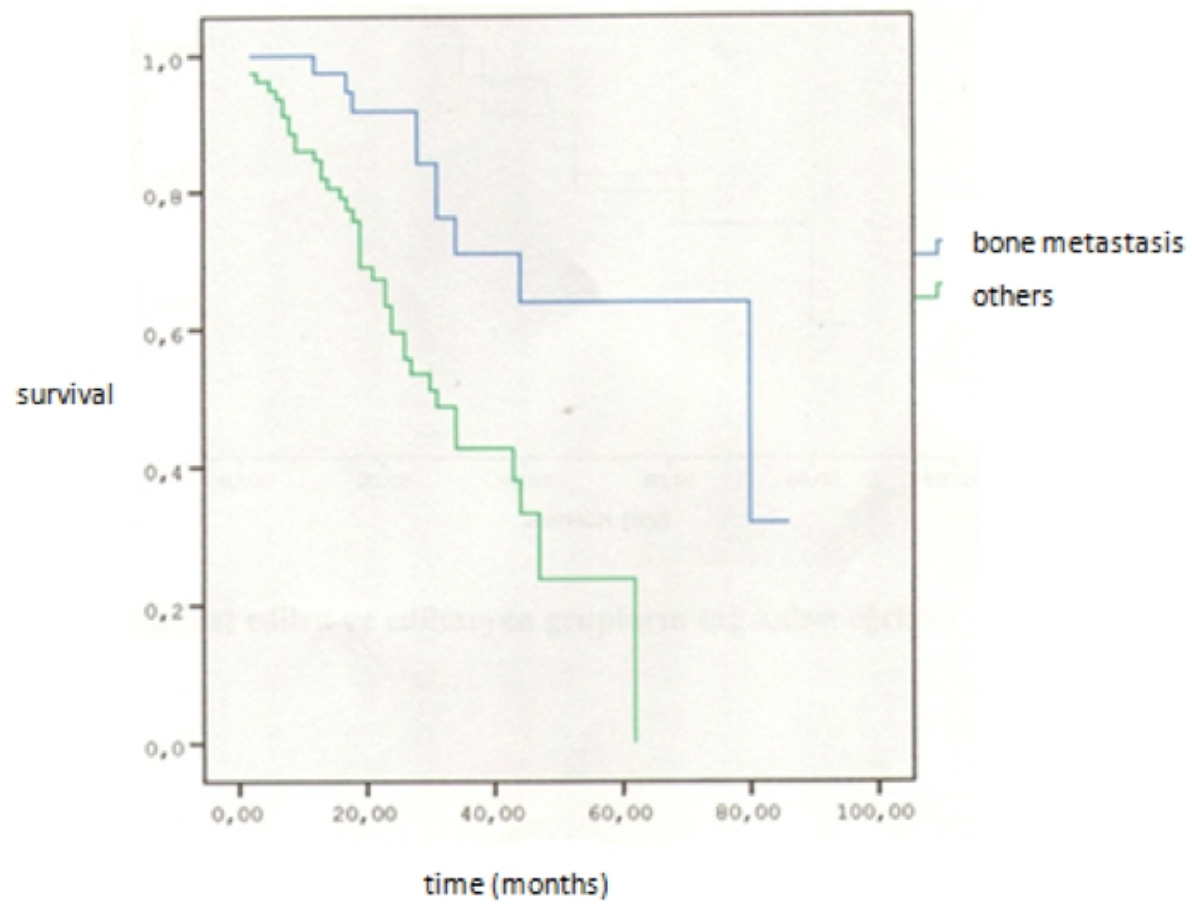

Fig. 1. Two-year Kaplan-Meier survival curves for patients with bone or other metastasis $(p=0.013)$

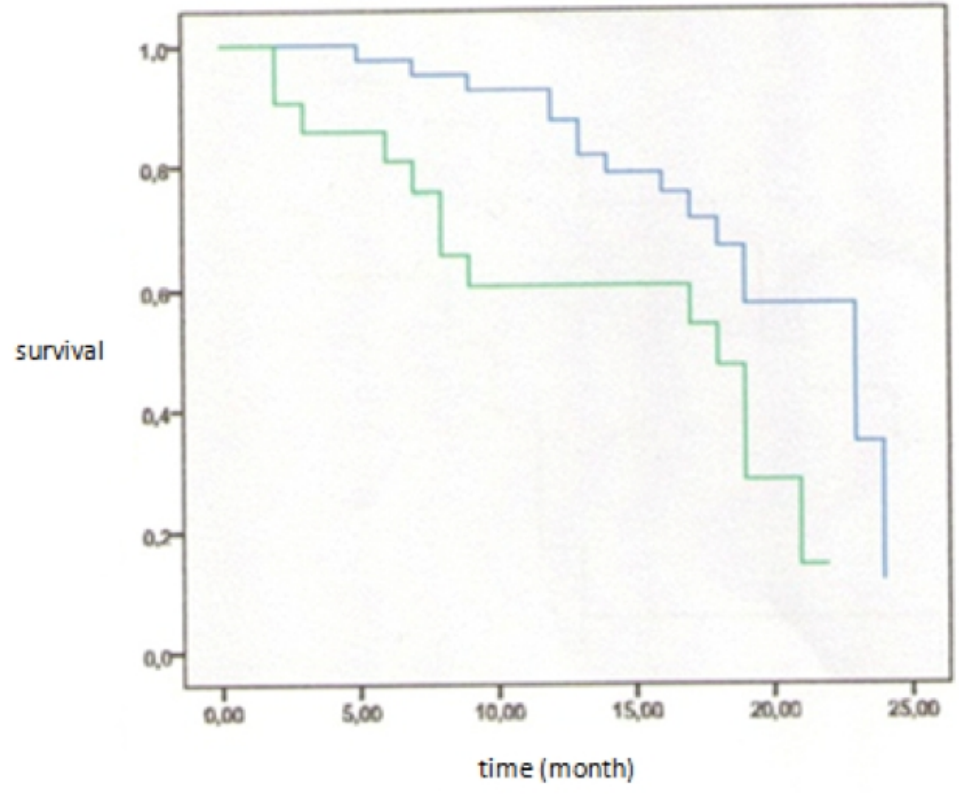

surgery

П $(+)$

$\Pi(-)$

Fig. 2. Two-year Kaplan-Meier survival curves in operation and non-operation groups $(p<0.001)$ 


\subsection{Discussion}

In this retrospective comparative study, we evaluated the impact of surgery in patients with stage IV breast cancer on survival and factors affecting the survival. We found that the median overal and two-year survival was significantly higher in the operation group than the group that did not undergo operation. The favorable effect of surgery was prominent in patients with visceral metastasis, while surgery had no effect on survival in patients with bone metastasis.

Haagensen and Stout published the criteria of inoperability for breast cancer in 1943 [2]. They stated that survival rates of patients they considered to be inoperable were very low and compared the survival rates of patients who were actually inoperable according to their criteria but underwent operations with those of other patients in other clinics followed up without operation, suggesting that operation was not beneficial. However, this study and its comparison methods are no longer valid. The criteria of inoperability suggested in their study, such as findings of oedema in the arms, supraclavicular lymph node metastasis, and inflammatory breast cancer are no longer regarded as appropriate.

The effect of surgical intervention for a primary tumour when distant metastasis, which is one of these criteria, is detected was first reported by Khan et al. in 2002 [3]. After their demonstration of a favourable effect of removal of the primary tumour with negative margins on survival, similarly positive results were reproduced in other clinical studies. Likewise, in the present study, the median survival of the groups that underwent surgery was 44 months, while that of the groups that did not was 34 months.

In some studies demonstrating that survival rates increase with removal of the primary tumour in patients who are at stage IV at the time of diagnosis, patients in the surgical treatment group were relatively younger and in better general condition and had merely bone metastasis or a limited metastatic burden. This led to the criticism that increased survival rates may also be attributed to factors other than surgery [4]. Similar to these studies, a difference was found between the two groups in the present study with respect to the number of metastatic regions and the type of metastasis. In the present study, the mean age of the operation group was $51.1 \pm 11.1$ years, whereas that of the non-operation group was $54.5 \pm 11.8$ years $(p=0.990)$. Patients with only bone metastasis accounted for $36.6 \%$ of those in the operation group, and patients with metastasis in a single region constituted $68.4 \%$ in this group.

In the analysis by Cady et al. of 622 patients with stage IV breast cancer with matched variables, although matching decreased or eliminated some increases in survival rates, when all types of metastasis were considered, the survival rate increased significantly in the operation group $(p=0.001)$ [5]. Again, when patients with only bone metastasis are matched, the survival rate has been shown to be increased $(p=0.003)$ [6]. In a study by Bafford et al. of 147 patients with stage IV breast cancer, in the evaluation made after the adjustment of variables such as age, number of metastatic regions, chemotherapy, and endocrine therapy, the median survival for the surgery group was 4.13 years, while that of the nonsurgical group was $2.36(p=0.003)$ [6]. These studies support the idea that surgical treatment increases survival rates, although patient selection also plays a part in this increase.

It is established that survival of patients with stage IV breast cancer has increased with time $[7,8]$. In the study by Dawood et al., which was a large series that included 15,438 patients, it 
was determined that survival rates have increased over time in patients newly diagnosed with stage IV breast cancer and that a higher tumour degree, increased age, not being married, hormone receptor-negative status, and lack of surgical treatment were associated with an unfavourable prognosis [8]. These studies demonstrated that surgical treatment of the primary tumour in patients with stage IV breast cancer has a positive impact on survival. However, what these studies lack is their inability to explain the reasons for this increase in survival. Similarly, in the present study, no factor could account for the increase in survival rate.

One possible mechanism is a decrease in the tumour burden. It was shown that in advanced-stage tumours such as colon, stomach, ovary, and renal cell carcinoma, resection makes a positive contribution to survival even when carried out for palliative reasons [9-16]. Based on this finding, it can be thought that in stage IV breast cancers, survival may be increased by a decrease in the tumour burden, enhancing the efficacy of chemotherapy and regulation of the immune system. In the present study, it was established that in patients with visceral metastasis there was a significant increase in two-year survival with surgery. A decrease in the tumour burden is the probable reason for this increase in survival.

It is known that breast cancer metastasises most commonly to the bone [4]. The survival rate in patients with only bone metastasis is higher than that in patients with extraosseous metastasis. Yamashita et al., reported that the median survival was 5 months after the appearance of extraosseous metastasis, while that in patients with only bone metastasis was 21.5 months [17]. Likewise, in the present study, the survival rate of patients with only bone metastasis was significantly higher than that of patients with extraosseous spread in both the operation and non-operation groups. Therefore, in patients with a slower clinical course, interventions for elimination of the primary tumour, which is the main source of circulating tumour cells, may be performed with lower morbidity and mortality.

Removal of the primary tumour may have an impact on survival through decreasing the number of circulating tumour cells. In many studies, it was demonstrated that tumour spread in breast cancer occurs in the very early period [18]. Braun et al. reported that of 4703 patients with breast cancer of stages I-III, the rate of bone marrow micrometastasis was $30.6 \%$. In addition, the presence of micrometastasis had a significant effect on both total survival and disease-free survival [18].

The present study had some limitations. Firstly, the study design was retrospective which limits control of some profounding factors. On the basis of the present findings, further prospective studies on the impact of surgical treatment in combination with or without systemic treatment in patients with stage IV breast cancer. Secondly, due to retrospective nature of the study and limited technical resources, histological findings such as HER2 status, Ki-67, histological grade, and nuclear grades are not complete for all patients, thus we could not report them. Furthermore since patients were given various types of medical treatment we did not analyze the treatment types and its effect on survival. It should be noted that these limitations could alter our finding that in some cases there is survival benefit from primary tumor removal. However, this topic has produced a number of reports and consistency is not common. Our study is valuable in that it will help clinicians and patients make difficult decisions in cases of metastatic breast cancer and provide basis for further controlled studies. 


\section{CONCLUSION}

In conclusion, breast cancer should be regarded as a systemic disease at the time of diagnosis. The surgical treatment has favorable effect on survival of patients with stage IV breast cancer, particularly those with only visceral metastasis. Thus surgical resection of primary tumor should be applied in stage IV breast cancer with only visceral metastasis along with systemic treatment to increase success rates. Further prospective and randomized studies are required to demonstrate this effect more clearly and to determine the patient groups that will benefit more from surgical treatment.

\section{CONSENT}

Informed consent was waived by the Institutional Ethics Committee of Hacettepe University for the retrospective design of the study.

\section{ETHICAL APPROVAL}

The study was approved by the Institutional Ethics Committee of Hacettepe University.

All authors hereby declare that all experiments have been examined and approved by the appropriate ethics committee and have therefore been performed in accordance with the ethical standards laid down in the 1964 Declaration of Helsinki.

\section{ACKNOWLEDGEMENTS}

The study had no funding support.

\section{COMPETING INTERESTS}

Authors declared that they have no competing interests.

\section{REFERENCES}

1. U.S. Cancer Statistics Working Group. United States Cancer Statistics: 1999-2009 Incidence and Mortality Web-based Report. Atlanta (GA): Department of Health and Human Services, Centers for Disease Control and Prevention, and National Cancer Institute; 2013. Accessed 12 June 2012. Available: http://www.cdc.gov/uscs.

2. Haagensen CD, Stout AP. Carcinoma of the breast. II-Criteria of operability. Ann Surg. 1943;118(6):1032-51.

3. Khan SA, Stewart AK, Morrow M. Does aggressive local therapy improve survival in metastatic breast cancer? Surgery. 2002;132(4):620-7.

4. Gonzalez-Angulo AM, Morales-Vasquez F, Hortobagyi GN. Overview of resistance to systemic therapy in patients with breast cancer. Adv Exp Med Biol. 2007;608:1-22.

5. Cady B, Nathan NR, Michaelson JS, Golshan M, Smith BL. Matched pair analyses of stage IV breast cancer with or without resection of primary breast site. Ann Surg Oncol. 2008;15(12):3384-95.

6. Bafford AC, Burstein HJ, Barkley CR, Smith BL, Lipsitz S, Iglehart JD, et al. Breast surgery in stage IV breast cancer: impact of staging and patient selection on overall survival. Breast Cancer Res Treat. 2009;115(1):7-12. 
7. Andre F, Slimane K, Bachelot T, Dunant A, Namer M, Barrelier A, et al. Breast cancer with synchronous metastases: trends in survival during a 14-year period. J Clin Oncol. 2004;22(16):3302-8.

8. Dawood S, Broglio K, Gonzalez-Angulo AM, Buzdar AU, Hortobagyi GN, Giordano $\mathrm{SH}$. Trends in survival over the past two decades among white and black patients with newly diagnosed stage IV breast cancer. J Clin Oncol. 2008;26(30):4891-8.

9. Cook AD, Single R, McCahill LE. Surgical resection of primary tumors in patients who present with stage IV colorectal cancer: an analysis of surveillance, epidemiology, and end results data, 1988 to 2000. Ann Surg Oncol. 2005;12(8):637-45.

10. Beham A, Rentsch M, Püllmann K, Mantouvalou L, Spatz H, Schlitt HJ, et al. Survival benefit in patients after palliative resection vs non-resection colon cancer surgery. World J Gastroenterol. 2006;12(41):6634-8.

11. Lin SZ, Tong HF, You T, Yu YJ, Wu WJ, Chen C, et al. Palliative gastrectomy and chemotherapy for stage IV gastric cancer. J Cancer Res Clin Oncol. 2008;134(2):18792.

12. Ouchi K, Sugawara T, Ono H, Fujiya T, Kamiyama Y, Kakugawa Y, et al. Therapeutic significance of palliative operations for gastric cancer for survival and quality of life. $J$ Surg Oncol. 1998;69(1):41-4.

13. Saidi RF, ReMine SG, Dudrick PS, Hanna NN. Is there a role for palliative gastrectomy in patients with stage IV gastric cancer? World J Surg. 2006;30(1):21-7.

14. Flanigan RC, Salmon SE, Blumenstein BA, Bearman SI, Roy V, McGrath PC, et al. Nephrectomy followed by interferon alfa-2b compared with interferon alfa-2 $b$ alone for metastatic renal-cell cancer. N Engl J Med. 2001;345(23):1655-9.

15. Todo $Y$, Sakuragi N, Oikawa M, Negishi H, Yamamoto R, Yoshiaki K, et al. Cytoreductive surgery combined with organ resection for advanced ovarian carcinoma. Int J Clin Oncol. 2003;8(2):90-6.

16. Aletti GD, Gallenberg MM, Cliby WA, Jatoi A, Hartmann LC. Current management strategies for ovarian cancer. Mayo Clin Proc. 2007;82(6):751-70.

17. Yamashita $\mathrm{K}$, Koyama $\mathrm{H}$, Inaji $\mathrm{H}$. Prognostic significance of bone metastasis from breast cancer. Clin Orthop Relat Res. 1995;312:89-94.

18. Braun S, Vogl FD, Naume B, Janni W, Osborne MP, Coombes RC, et al. A pooled analysis of bone marrow micrometastasis in breast cancer. $\mathrm{N}$ Engl $\mathrm{J}$ Med. 2005;353(8):793-802.

(C) 2013 Erol et al.; This is an Open Access article distributed under the terms of the Creative Commons Attribution License (http://creativecommons.org/licenses/by/3.0), which permits unrestricted use, distribution, and reproduction in any medium, provided the original work is properly cited.

Peer-review history:

The peer review history for this paper can be accessed here: http://www.sciencedomain.org/review-history.php?iid=205\&id=12\&aid=1610 